\title{
Endophytic and Epiphytic Phyllosphere Fungal Communities Are Shaped by Different Environmental Factors in a Mediterranean Ecosystem
}

\author{
Teresa Gomes $^{1,2}$ • José Alberto Pereira ${ }^{1} \cdot$ Jacinto Benhadi $^{1} \cdot$ Teresa Lino-Neto $^{2} \cdot$ Paula Baptista $^{1}$ (D)
}

Received: 18 November 2017 / Accepted: 13 February 2018

(C) Springer Science+Business Media, LLC, part of Springer Nature 2018

\begin{abstract}
The diversity and factors influencing fungal assemblages in phyllosphere of Mediterranean tree species have been barely studied, especially when endophytic and epiphytic communities are simultaneously considered. In this work, the endophytic and epiphytic fungal communities from olive tree phyllosphere were studied. This tree species is natural from the Mediterranean region and adapted to grow under adverse climatic conditions. The main objectives were to determine whether there are differences between both fungal communities and to examine whether different abiotic (climate-related) and biotic (plant organs) factors play a pivotal role in structuring these communities. Both communities differed in size and composition, with epiphytic community being richer and more abundant, displaying also a dominance of melanized fungi. Season was the major driver of community composition, especially of epiphytes. Other drivers shaping epiphytes were wind speed and temperature, while plant organ, rainfall, and temperature were the major drivers for endophytic composition. In contrast, canopy orientation caused slight variations in community composition of fungi, but with distinct effects in spring and autumn seasons. In conclusion, epiphytic and endophytic communities are not driven by the same factors. Several sources of variation undergo complex interactions to form and maintain phyllosphere fungal community in Mediterranean climates. Climatic parameters have influence on these fungal communities, suggesting that they are likely to be affected by climate changes in a near future.
\end{abstract}

Keywords Climatic factors $\cdot$ Fungal community structure $\cdot$ Olea europaea L. · Organ specificity $\cdot$ Seasonal patterns

\section{Introduction}

Phyllosphere is the aboveground component of plants, supporting a diverse community of microorganisms that live both within (as endophytes) and/or on the surface (as epiphytes) of plant tissues [1]. Phyllosphere fungi have been considered as determinant factors for plant health and productivity [2], but different drivers that shape these fungal communities on woody plants remain unclear, especially in the

Electronic supplementary material The online version of this article (https://doi.org/10.1007/s00248-018-1161-9) contains supplementary material, which is available to authorized users.

Paula Baptista

pbaptista@ipb.pt

1 School of Agriculture - Polytechnic Institute of Bragança, CIMO, Campus Sta Apolónia, 5300-253 Bragança, Portugal

2 Biosystems \& Integrative Sciences Institute (BioISI), Plant Functional Biology Center (CBFP), University of Minho, Campus de Gualtar, 4710-057 Braga, Portugal
Mediterranean region [3]. Reports are only limited to Mediterranean oak forests (e.g., [3-7]), pine stands (e.g., [6, 8]), and poplar plantations (e.g., [9]). Some of these studies tried to determine the factors that affect community composition of endophytic fungi, such as climatic variables [6, 8], type of plant organs [7-9], and seasonality [4]. However, the results of these studies were often contradictory. Climatic variables, such as average temperature, water availability, and solar radiation, were reported as drivers of endophytic fungal community composition inhabiting twigs and needles of Pinus halepensis [8]. In other pine plantations (P. sylvestris, $P$. nigra, and P. pinaster) and native oak forests (Quercus pyrenaica), the influence of climatic variables was not clearly understood [6]. Previous studies have reported that the composition of fungal endophytes was different in leaves and twigs of Q. cerris and Q. pubescens [7], while in other perennial plants, there were no obvious evidences to prove tissue/ organ specificity of fungal endophytes [9]. Furthermore, most studies have focused exclusively on endophytic fungi and have not considered epiphytic community, even though epiphytes may live less than a millimeter apart and distinction 
between both life forms may not be clear. In woody plants, fungal endophytes are transmitted horizontally [10], so presumably most of them grow on leaf surface before penetrating into plant tissues [11]. However, the surface and internal tissues of these aboveground organs represent two different habitats. Epiphytes are exposed to numerous external environmental stress factors, such as temperature, humidity, and solar radiation. On the other hand, endophytes have a more sheltered habitat but are challenged by plant defense reactions [12]. Different stress factors faced by epiphytes and endophytes might have important effects on their community composition. The only studies that compared epiphytic and endophytic fungal communities on leaves of deciduous shrubs [13] or woody plants, such as Coffea arabica [14] and Camellia japonica [15], reported that they have distinct communities.

Mediterranean-type ecosystems, like those found in the Iberian Peninsula, are frequently challenged by several stress factors, such as temperature, rainfall, and UV radiation. Therefore, a better knowledge of epi- and endophytic fungal communities that thrive in phyllosphere of Mediterranean environments is needed. This would be useful for getting a better understanding of factors that influence fungal composition and structure, in order to predict their response to climate change. In this study, we determined the influence of some climatic variables (i.e., rainfall, temperature, relative humidity, and wind speed), seasons (spring vs. autumn), cardinal direction (north vs. south), and plant organ type (leaf vs. twig) on the composition of both epi- and endophytic fungi present on phyllosphere of olive trees. Olea europaea L. is an ancient drought-tolerant crop, which has significant ecological and socioeconomic importance for Mediterranean countries [16]. By identifying factors that play a dominant role in the structure of phyllospheric fungal communities in Mediterranean ecosystems, novel strategies could be developed for mitigating the impacts of climate change and for improving agriculture.

\section{Material and Methods}

\section{Study Site and Sample Collection}

Samples were collected from three olive orchards located in Mirandela, Northeast of Portugal [N 41 32.593'; W $07^{\circ}$ 07.445' (orchard 1), N 41 $32.756^{\prime}$; W 07 07.590' (orchard 2) and $\mathrm{N} 41^{\circ} 29.490^{\prime} ; \mathrm{W} 07^{\circ} 15.413^{\prime}$ (orchard 3)]. This is a mountainous region, with altitudes ranging between 300 and $500 \mathrm{~m}$, displaying a Mediterranean climate, with cold and rainy winters and long, hot, and dry summers. The average annual rainfall in Mirandela ranged from 500 to $700 \mathrm{~mm}$, mainly occurring between October and February, and annual average temperature ranged from 3 to $26^{\circ} \mathrm{C}$ [17]. The selected orchards were managed by following integrated production guidelines [18]. Each orchard included Portuguese olive cultivars, wherein trees were planted at $7 \times 7 \mathrm{~m}$ spacing. In each orchard, olive branches were collected from 21 randomly chosen trees, in two cardinal directions (north and south) at the operator's height (one sample from north and other from south $\times 21$ trees $\times 3$ orchards $=126$ branches). The same trees were sampled at two different periods, i.e., in autumn 2013 (from October to November) and spring 2014 (from March to May).

\section{Climatic Data}

During the surveyed period, meteorological data was collected at a weather station located near olive orchards at Mirandela ( $\mathrm{N} 41^{\circ}$ 43.333'; W 07 21.944, elevation: 357 m). Cumulative rainfall, average daily mean temperature, relative humidity (maximum and minimum), and wind speed were determined at $3,5,10$, and 20 days before sampling dates.

\section{Fungal Isolation and Identification}

From each branch, five segments of twigs and leaves were randomly selected and used to isolate epiphytes and endophytes. For isolating fungal epiphytes, around $1 \mathrm{~g}$ of leaves and twigs was separately added to $9 \mathrm{~mL}$ of sterile potassium phosphate buffer pH 7.0 (8 g/L NaCl; $0.20 \mathrm{~g} / \mathrm{L} \mathrm{KCl} ; 1.4 \mathrm{~g} / \mathrm{L} \mathrm{Na}_{2} \mathrm{HPO}_{4}$; $0.24 \mathrm{~g} / \mathrm{L} \mathrm{KH}_{2} \mathrm{PO}_{4}$ ). This suspension was placed on a rotary shaker (200 rpm) for $60 \mathrm{~min}$, at room temperature, to dislodge microorganisms from the plant surface. Aliquots of $1 \mathrm{~mL}$ of the resulting microbial suspensions were separately plated in triplicate on Potato Dextrose Agar (PDA, Difco) and Plate Count Agar (PCA, Himedia) media, supplemented with $0.01 \%(w / v)$ chloramphenicol (Oxoid). Plates were incubated at $25 \pm 2{ }^{\circ} \mathrm{C}$ in the dark and were observed daily for microbial growth and colony counting. Results of epiphytes were expressed as log CFU/ $\mathrm{cm}^{2}$, i.e., the number of individual colonies of fungi adhered to the surface of leaf/twig. An ellipse equation $(A=\pi a b \times 2)$ was used for estimating leaf surface. A cylinder equation $(A=$ $2 \pi r h+2 \pi r^{2}$ ) was used for determining twig surface. In these equations, $A$ is the area, $a$ and $b$ are the longitudinal and transverse axes of leaf, respectively, and $r$ and $h$ are radius and height of twig segments, respectively. The average area of leaf and twig was $13.5 \pm 1.3 \mathrm{~cm}^{2}$ and $11.4 \pm 0.1 \mathrm{~cm}^{2}$, respectively.

Endophytic fungi were isolated from the same plant fragments used to isolate epiphytes. After removing epiphytes by surface disinfection through a procedure described by Martins et al. [19], each leaf or twig was cut into segments (4-5 mm for twigs and ca. $5 \times 5 \mathrm{~mm}$ for leaves), which were transferred into the same culture media used to isolate epiphytes. In total, 7440 plant tissue segments were inoculated for each season (spring and autumn). To validate surface sterilization procedure, the surface of sterilized plant tissues was imprinted onto PDA and PCA media.

Fungal colonies were subcultured on fresh medium until pure epi/endophytic cultures were obtained. Each fungal 
colony was further identified by morphological and molecular approaches. Isolates were first grouped, according to their morphological similarity (colony morphology, hyphae, spores, and reproductive structures). One representative isolate of each morphotype was selected for molecular identification, using internal transcribed spacer (ITS) region of the nuclear ribosomal DNA (rDNA). Total genomic DNA was extracted from harvested mycelia/spores using REDExtract-N-Amp ${ }^{\text {TM }}$ Plant PCR kit (Sigma, Poole, UK). The ITS region (ITS1, 5.8S, ITS2) was amplified using ITS1/ITS4 or ITS5/ITS4 primer sets [20] according to a PCR protocol previously described by Oliveira et al. [21]. The amplified products $(\sim 650 \mathrm{bp})$ were purified and sequenced using Macrogen Inc. (Seoul, South Korea) services. The obtained DNA sequences were analyzed with DNASTAR v.2.58 software, and fungal identification was performed using the NCBI database (http://www.ncbi.nlm.nih. gov) and BLAST algorithm. Blast results were sorted according to higher identity score and lowest E-value. For sequence identities $>98 \%$, the genus and species were accepted; for sequence identities between $95 \%$ and $97 \%$, only the genus was accepted; and for sequence identities $<95 \%$, isolates were labeled as "unknown" fungi. The sequences obtained are available at GenBank with the following accession numbers: KU324941-KU325040; KU325041KU325240; KU325241-KU325457; KT804020-KT804039; KT804040-KT804069; KT804070-KT804119; KT804120KT804149. Pure cultures of each identified isolate were preserved and deposited in the culture collection of the Polytechnic Institute of Bragança (School of Agriculture).

\section{Diversity of Fungal Endophytes and Epiphytes}

For the following analysis, only those OTUs identified up to the genus were considered, being excluded the "unknown fungi." Diversity of fungal epiphytes and endophytes was determined in tissue samples (leaf, twig, and total), oriented in a north-south position for each season (spring and autumn). Diversity was assessed by evaluating the abundance (average number of isolates per tree) and richness (average number of operational taxonomic units per tree), and by computing Simpson's Reciprocal Index (1/D) and Shannon-Wiener $(\mathrm{H})$ diversity indices with Species Diversity and Richness v. 4.0 [22]. All diversity indices and estimators are presented as the mean of replicates (= tree), displaying respective SE values or total number (the values of all samples lumped together). To determine differences among means, an analysis of variance (ANOVA) with SPSS v.18 was performed. Averages were then compared using Tukey's test $(p<0.05)$. Species-richness within tissue samples was also estimated by using species accumulation curves, which were calculated using sample-based rarefaction index (Mao Tau) and EstimateS v. 9.1.0 [23], using 1000 runs of bootstrapping with replacement.

\section{Data Analysis}

Both univariate and multivariate methods were used to identify which climatic or biological factors may drive the structure and composition of epiphytic and endophytic fungal community. For each fungal community (endophytic or epiphytic), mean fungal abundance, richness, and Simpson's Reciprocal Index diversity were modeled separately by using R software [24]. Organ (leaf vs. twig), cardinal direction (north vs. south), and season (spring vs. autumn) were considered as explanatory variables. Thus, the full linear model for each independent variable was as follows:

$$
\begin{aligned}
Y_{i}= & \alpha+\beta_{1} \times \text { Organ }_{i}+\beta_{2} \times \text { Cardinal direction }_{i}+\beta_{3} \\
& \times \text { Season }_{i}+\varepsilon_{i} \quad \text { where } \varepsilon_{i} \sim N(0,2)
\end{aligned}
$$

For each response, model selection followed Akaike's Information Criterion (AIC), according to Zuur et al. [25]. Then, the model was validated by plotting the fitted values vs. residuals, checking for non-linear patterns. For the endophyte community, an interaction term had to be included between organ and season in the diversity model. The optimal model for each response and community remained as follows:

Endophytic community: Abundance $_{i}=\alpha+\beta_{1} \times \operatorname{Organ}_{i}+\varepsilon_{i}$

$$
\begin{aligned}
& \text { Richness }_{i}=\alpha+\beta_{1} \times \text { Organ }_{i}+\beta_{2} \times \text { Season }_{i}+\varepsilon_{i} \\
& \text { Divsersity }_{i}=\alpha+\beta_{1} \times \text { Organ }_{i}+\beta_{2} \times \text { Season }_{i}+\beta_{3} \times \text { Organ }_{i}: \text { Season }_{i}+\varepsilon_{i}
\end{aligned}
$$

Epiphytic community: Abundance $_{i}=\alpha+\beta_{1} \times$ Season $_{i}+\varepsilon_{i}$

$$
\begin{aligned}
& \text { Richness }_{i}=\alpha+\beta_{1} \times \text { Season }_{i}+\varepsilon_{i} \\
& \text { Divsersity }_{i}=\alpha+\beta_{1} \times \text { Cardinal diretion }_{i}+\beta_{2} \times \text { Season }_{i}+\varepsilon_{i}
\end{aligned}
$$

Secondly, non-metric multidimensional scaling (NMDS) was carried out with two similarity indexes, i.e., Jaccard's and Bray-Curtis, in order to assess variability of endophytic and epiphytic assemblages with respect to season (autumn vs. 
spring), cardinal direction (north vs. south) and plant organ (leaf vs. twig). Both similarity indexes provided different types of information. Jaccard's similarity index only compares the presence or absence of fungal taxa among samples, without considering species abundance [26]. On the other hand, Bray-Curtis's similarity index compares the presence or absence of fungal taxa as well as species abundance among samples. This coefficient ignores cases in which species are absent in both community samples and is strongly influenced by abundant species [27]. Kruskal's stress was used to estimate model's goodness-of-fit (commonly acceptable when is $<0.2$ ). A one-way analysis of similarity (ANOSIM) was used to determine significant differences between fungal community groupings obtained in NMDS ordination, using Bray-Curtis distance matrices. ANOSIM generates a $p$ value (significant level below to 0.05 ) and $R$ value (gives the degree of discrimination between groups), which ranges from 0 (indistinguishable) to 1 (completely dissimilar) [28]. All these analyses were performed using Community Analysis Package v. 4.0 [29].

To corroborate the obtained results, a "cross-table" multivariate analysis (co-inertia analysis, CIA) was performed using the $\mathrm{R}$ software. This analysis aims to find a co-structure between two sets of variables that are linked by the same individuals, where the resulting sample scores the most covariant [30]. The co-inertia function from the "ade4" package in $\mathrm{R}$ was used to perform the analyses, and the table.value function from the same package was used to visualize the results.

Causes of variation in endophytic and epiphytic fungal assemblages due to climatic factors (i.e., mean temperature; maximum and minimum relative humidity; total rainfall and wind speed) were also investigated using distance-based linear models (DistLM) [31]. This analysis was performed on climatic data, obtained on 3, 5, 10 , and 20 days before fungal sampling, which were individually used or clustered together (for each climatic variable, days were lumped together). Similarity matrices were generated from square root transformed data of fungal abundance using the Bray-Curtis method and climatic data were $\log$ transformed $[\log (x+1)]$ to normalize their distribution. A marginal test was firstly performed, where the individual climatic variables were separately fitted to assess their relationship with fungal data. Using step-wise selection procedure and AIC, a sequential test was performed to identify the subset of climatic variables that best predicts the observed structural pattern of fungal community. Both marginal and sequential tests were undertaken with 999 permutations using PRIMER 7.0 [28].

\section{Results}

\section{Endophytic and Epiphytic Fungal Communities Differ in Diversity and Composition}

A total of 290 fungal operational taxonomic units (OTUs) were isolated from all plant tissues surveyed in both seasons (autumn and spring). Compared to endophytes (125 OTUs), epiphytes had greater diversity (242 OTUs; Table S1). Despite having high diversity, species accumulation curves did not reach an asymptote (Fig. 1a), indicating that more species
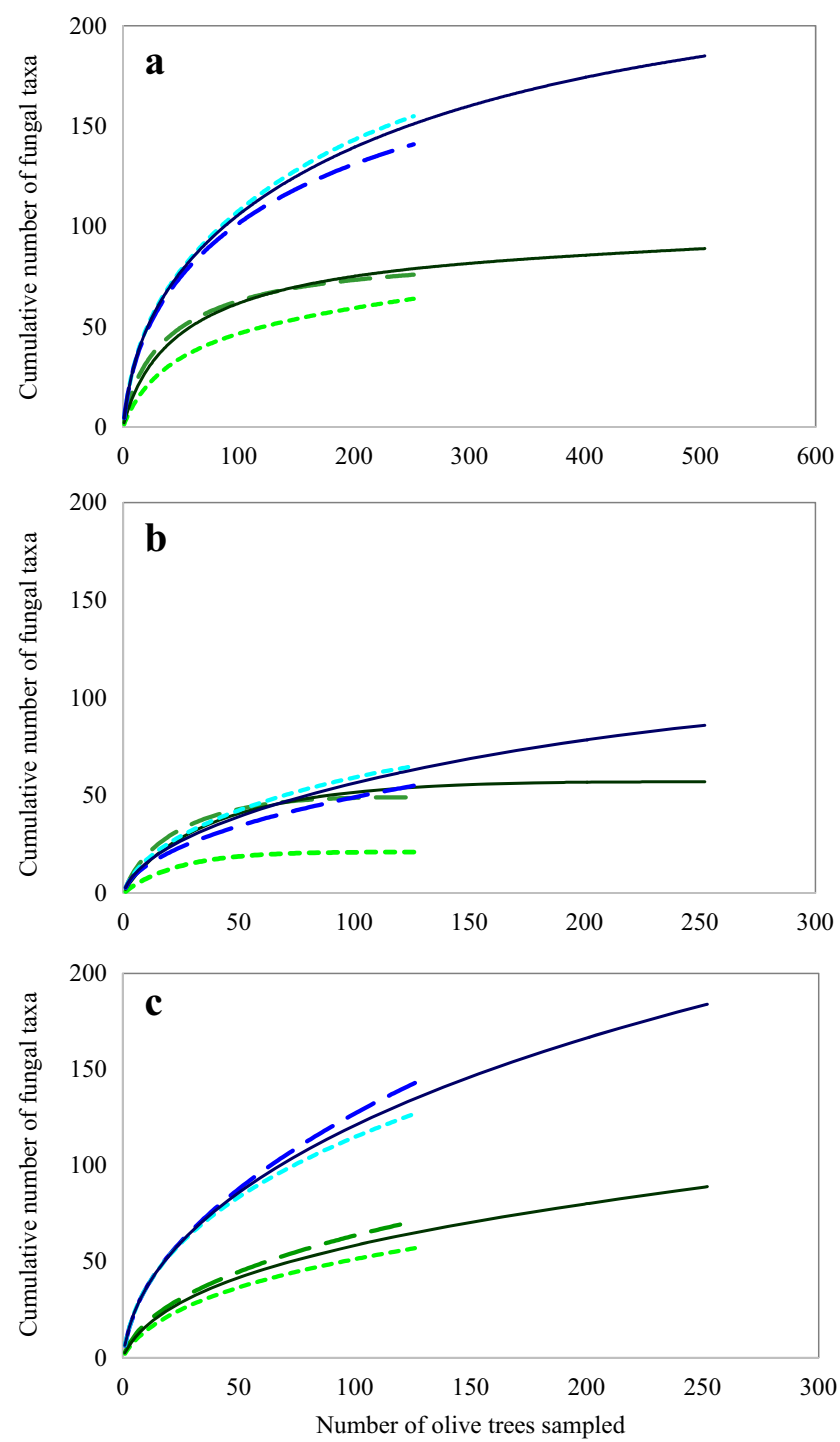

—-Endophytic Twigs $---\cdot$ Endophytic Leaves —-Total Endophytic
- Epiphytic Twigs --- Epiphytic Leaves - Total Epiphytic

Fig. 1 Species accumulation curves of fungal endophytes and epiphytes isolated from each plant tissue (leaves and twigs) and from whole plant (total), collected from 63 olive trees (Olea europaea L.), in both seasons (a), autumn (b), and spring (c) 
would be isolated with additional sampling. For endophytes, 135 OTUs would be expected (instead of the observed 125), and for epiphytes, 254 OTUs were expected instead of the observed 242. Both Simpson and Shannon-Winer indices were similar between endophytic and epiphytic communities ( 3.5 vs. 3.6 and 0.7 vs. 1.1, respectively), despite the average number of epiphytic fungal OTUs per tree being significantly higher $(p<0.001)$ than endophytic (5.4 vs. 2.9) (Table S1). Moreover, epiphytes were found to be more abundant than endophytes. Overall, the average number of epiphytic isolates per tree was up to 2.4-fold significantly higher $(p<0.001)$ than that of the endophytes (Table S1).

Endophytic and epiphytic fungal communities were significantly different in terms of species composition (ANOSIM, $R=0.465, p=0.001$ ) and were distinguished from each other by their most common families (Fig. 2). Within epiphytic communities, most abundant families were Davidiellaceae, Psathyrellaceae, Pleosporaceae, Nectriaceae, and Trichocomaceae. Within endophytic communities, the most frequently isolated fungal OTUs belonged to the following families: Leptosphaeriaceae, Pleosporaceae, Pyronemataceae, Trichocomaceae, and Pezizaceae. Most OTUs identified in this study were either exclusively endophytic (48) or epiphytic (165), and only 77 OTUs were found in both fungal communities (Fig. S1a). ANOSIM analysis indicated that dissimilarity between the composition of endophytic and epiphytic fungal communities was greater in spring $(R=0.976 ; p=0.001)$ than in autumn $(R=0.539 ; p=0.001)$, as well as in olive tree twigs $(R=0.542 ; p=0.001)$ than in leaves $(R=0.303 ; p=0.001)$. In contrast, dissimilarity between these two fungal communities was almost the same in south $(R=0.404 ; p=0.001)$ and in north directions $(R=0.388 ; p=0.001)$. These results were corroborated by NMDS ordination, in which a clear differentiation of endophytic from epiphytic fungal communities was evident for different seasons and olive tree organs, but not for cardinal direction (Fig. 3).

\section{Diversity and Composition of Phyllosphere Fungal Community Depends on the Season}

Fungal diversity (i.e., species richness and 1/D) of endophytes and epiphytes was significantly $(p<0.001)$ higher in spring than in autumn (Fig. 4). Since higher diversity was found in spring season, fungal surveys should to be carried out with greater sampling effort in spring than in autumn. For both fungal communities (especially for endophytes), overall species accumulation curves obtained in autumn appeared to be more close to an asymptote than the curves obtained in spring (Fig. 1). For epiphytic community, there was a 2.9-fold significant increase $(p<0.001)$ on fungal abundance (no. of isolates) from autumn to spring (Fig. 4b). a

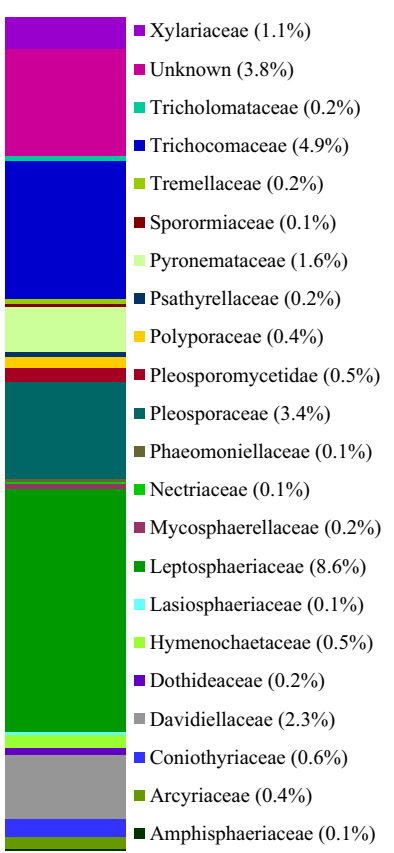

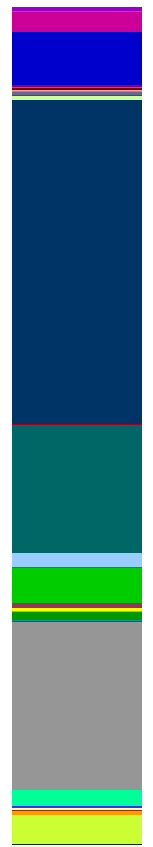

Frequency of occurrence (\%)

Epiphytes

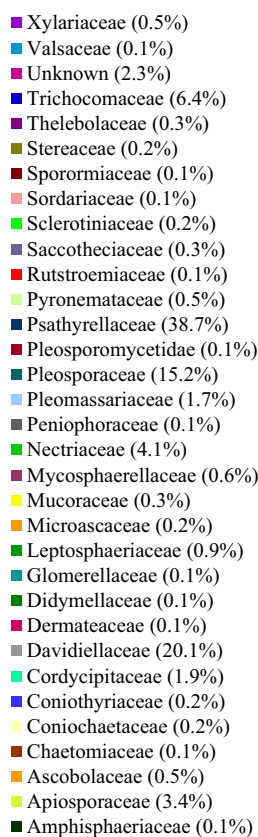

Valsaceae $(0.1 \%)$

Stereaceae $(0.2 \%)$

Sporormiaceae $(0.1 \%)$

Sordariaceae $(0.1 \%)$

Saccotheciaceae $(0.3 \%)$

Rutstroemiaceae $(0.1 \%)$

Pyronemataceae $(0.5 \%)$

Pleosporomycetidae $(0.1 \%)$

Pleosporaceae $(15.2 \%)$

Pleomassariaceae $(1.7 \%)$

Peniophoraceae $(0.1 \%)$

Nectriaceae $(4.1 \%)$

Mucoraceae $(0.3 \%)$

Microascaceae $(0.2 \%)$

Leptosphaeriaceae $(0.9 \%)$

merellaceae $(0.1 \%)$

Didymellaceae $(0.1 \%)$

Davidiellaceae $(20.1 \%)$

Cordycipitaceae $(1.9 \%)$

Coniothyriaceae $(0.2 \%)$

Coniochaetaceae $(0.2 \%)$

Ascobolaceae $(0.5 \%)$

Amphisphaeriaceae $(0.1 \%)$ b
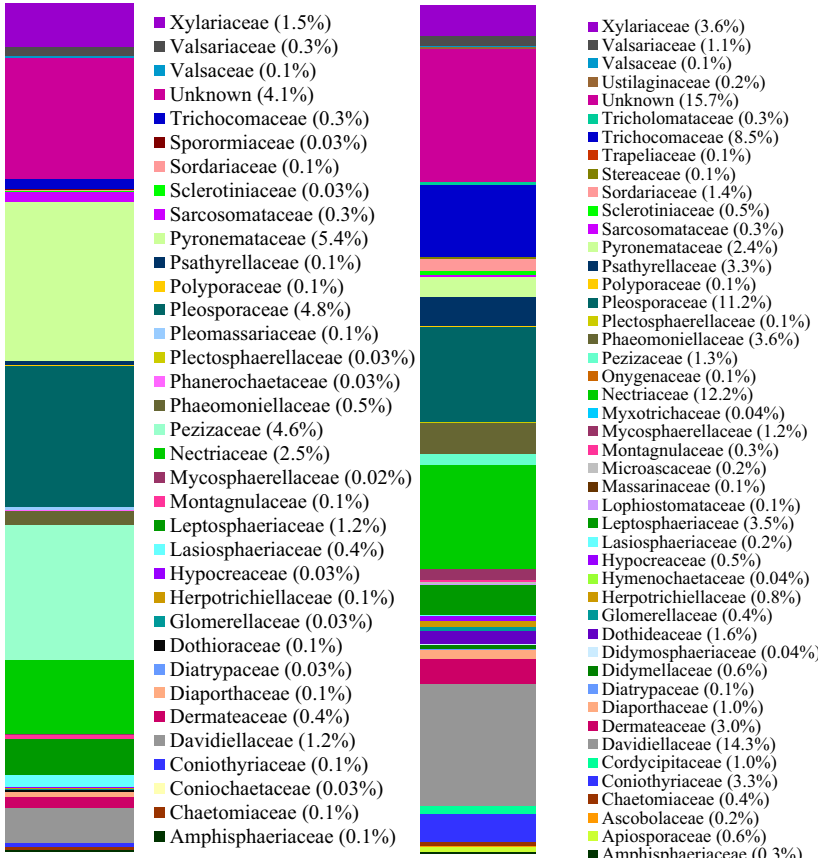

Frequency of colonization (\%)

Endophytes
Frequency of occurrence (\%)

Epiphytes

Fig. 2 Frequency of fungal families of endophytes and epiphytes present in Olea europaea L. tissues (twigs and leaves) surveyed either in autumn (a) or spring (b) 
Fig. 3 Non-metric multidimensional scale (NMDS) plots corresponding to the clustering of endophytic $(\Delta)$ and epiphytic $(\mathrm{O})$ communities grouped by (a) season (autumn and spring), (b) olive tree organ (leaves and twigs), and (c) cardinal direction (north and south). Cluster analysis was performed with two different community similarity measures, namely, Jaccard's index (binary data) and Bray-Curtis coefficient (raw abundance data). Kruskal's stress values less than 0.2 represent good ordination plots
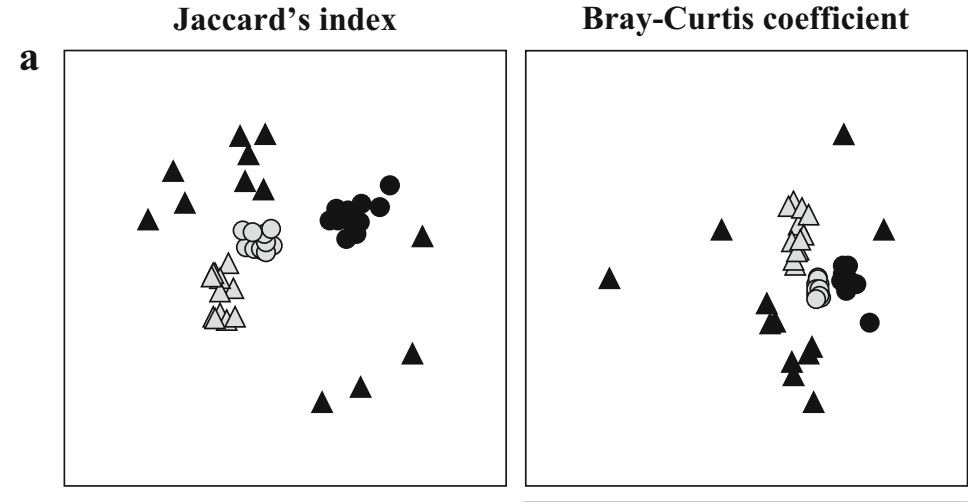

Autumn

b
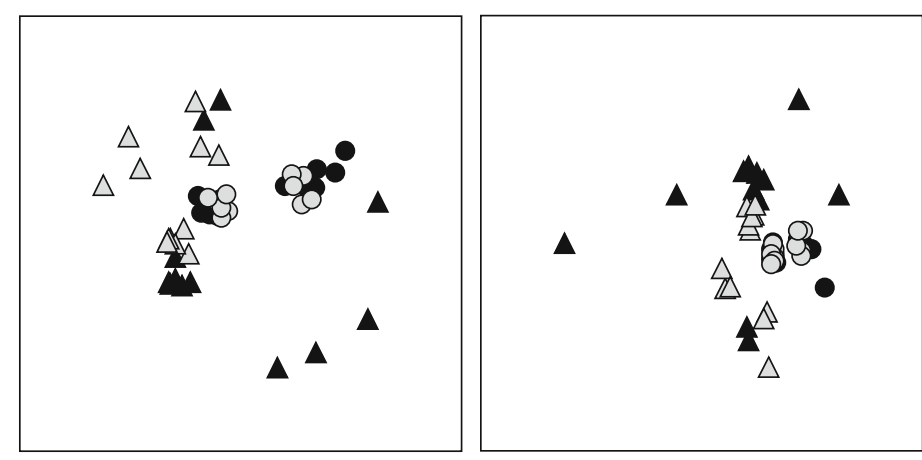

Leaves

c

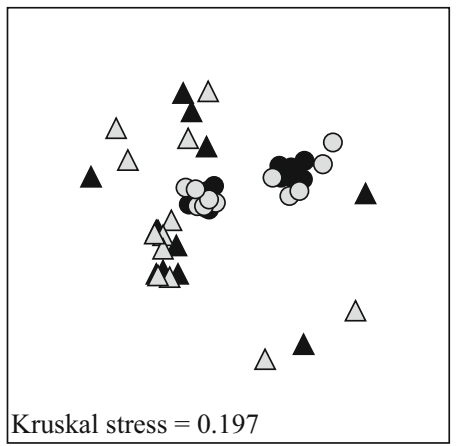

$\triangle$ Endophytes

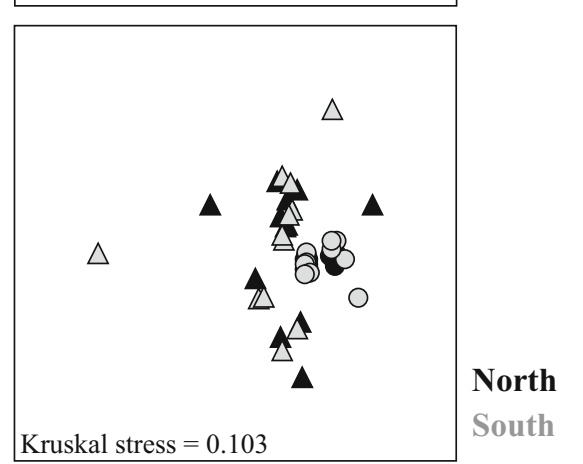

$\bigcirc$ Epiphytes Spring
NMDS plots, based on Jaccard's and Bray-Curtis indexes, and ANOSIM analysis showed that whole fungal communities composition differ significantly between seasons $(R=0.674$, $p=0.001)$. This dissimilarity was greater for epiphytes $(R=$ $0.940 ; p=0.001)$ than for endophytes $(R=0.540 ; p=0.001)$. In fact, only 44 species out of the 290 recovered in this study were isolated in both seasons; 164 species were isolated only in spring and 82 were recovered only in autumn (Fig. S1b). There was also some variation in the most abundant families found in both seasons, which accounted for differences in the composition of fungal communities in spring and autumn (Fig. 2). For example, endophytic OTUs belonging to families Leptosphaeriaceae and Trichocomaceae were preferentially isolated in autumn, while endophytes from Pyronemataceae, Pleosporaceae, and Pezizaceae families were more frequent and/or were exclusively isolated (e.g., Pezizaceae) in spring. Epiphytes belonging to Psathyrellaceae were more frequently isolated in autumn, whereas in spring were those from Davidiellaceae family.

\section{Leaves and Twigs Have Similar Microbiota, but Diversity of Fungal Endophyte Communities Is Different}

The total number of epiphytic OTUs found in twigs was higher than that in leaves (185 vs. 163 , Table S1), but there were no significant differences in fungal abundance (no. of isolates), richness, and diversity (1/D) in both the organs (Fig. 4b). This pattern was observed in either autumn or spring (Table S1). In contrast, within endophytic communities, twigs exhibited significantly $(p<0.001)$ higher diversity (up to 1.1 fold), richness (up to 1.4-fold), and fungal abundance (up to 3.1-fold) as compared to leaves (Fig. 4a; Table S1). This pattern was particularly observed in autumn season, where an increase 


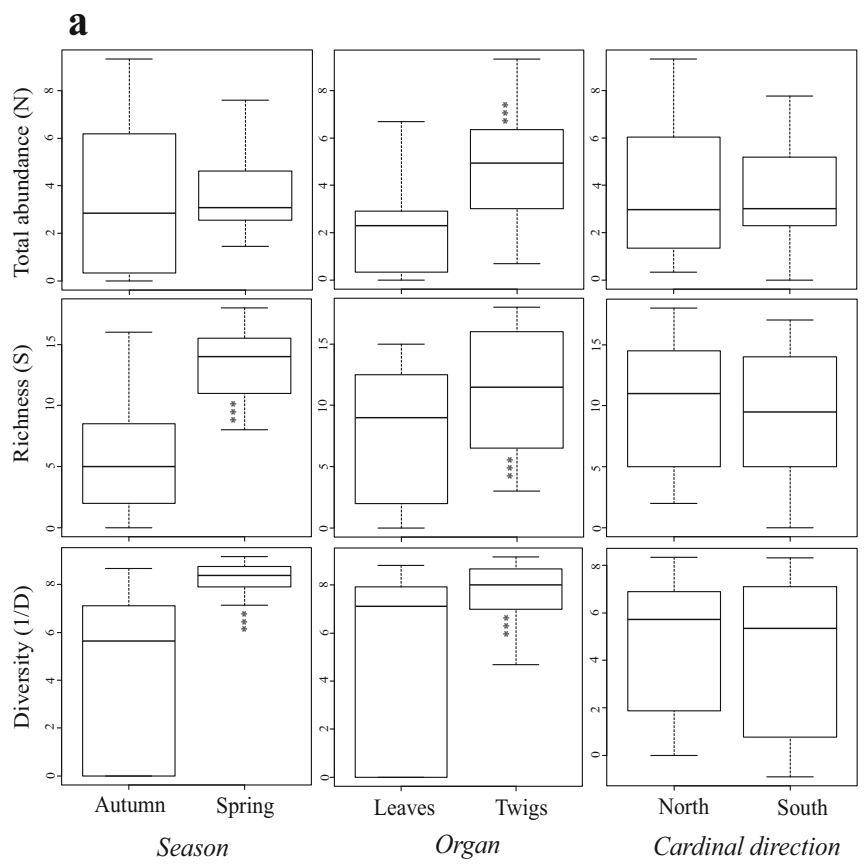

Fig. 4 Multiple linear regression regarding the total abundance $(\mathrm{N})$, richness (S) and diversity (1/D), within endophytic (a) and epiphytic (b) fungal communities in order to evaluate their significance with season

on diversity (up to 2.7- and 1.1-fold for $\mathrm{H}$ and 1/D, respectively), richness (up to 2.8-fold), and endophyte abundance (up to 4.4-fold) was significantly greater $(p<0.001)$ in twigs than in leaves. In spring, only endophytic richness of twigs was 1.4fold significantly higher $(p<0.001)$ than that of leaves.

According to the NMDS plots (Fig. 3b) and ANOSIM analysis, fungal community composition of twigs was very similar to leaves either for endophytes ( $R=0.090, p=0.049$ ) or epiphytes ( $R=0.133, p=0.057)$. Out of total fungal taxa, around $43 \%$ was shared between both organs of olive trees (Fig. S1c).

\section{Phyllosphere Fungal Communities from North and South Organs Did Not Differ in Diversity and Composition, but Depends on Season}

When organ tissues were taken from both cardinal directions (north and south), both endophytic and epiphytic communities showed no significant differences with respect to fungal abundance, diversity and richness (Fig. 4). Differences became evident when considering samples collected in different seasons (Table S2). In spring, the abundance of fungal endophytes was higher in north than in south direction (up to 1.2fold; $p<0.05)$. In autumn, diversity $(\mathrm{H})$, richness and abundance of fungal epiphytes was higher in north than in south direction (up to 1.8 -fold; $p<0.01$ ) (Table S2).

According to the NMDS plots (Fig. 3c) and ANOSIM analysis, a high degree of overlap of fungal taxa was observed for epiphytes $(R=0.132, p=0.053)$ and especially for endophytes $(R=0.042, p=0.882)$ in north and south directions.

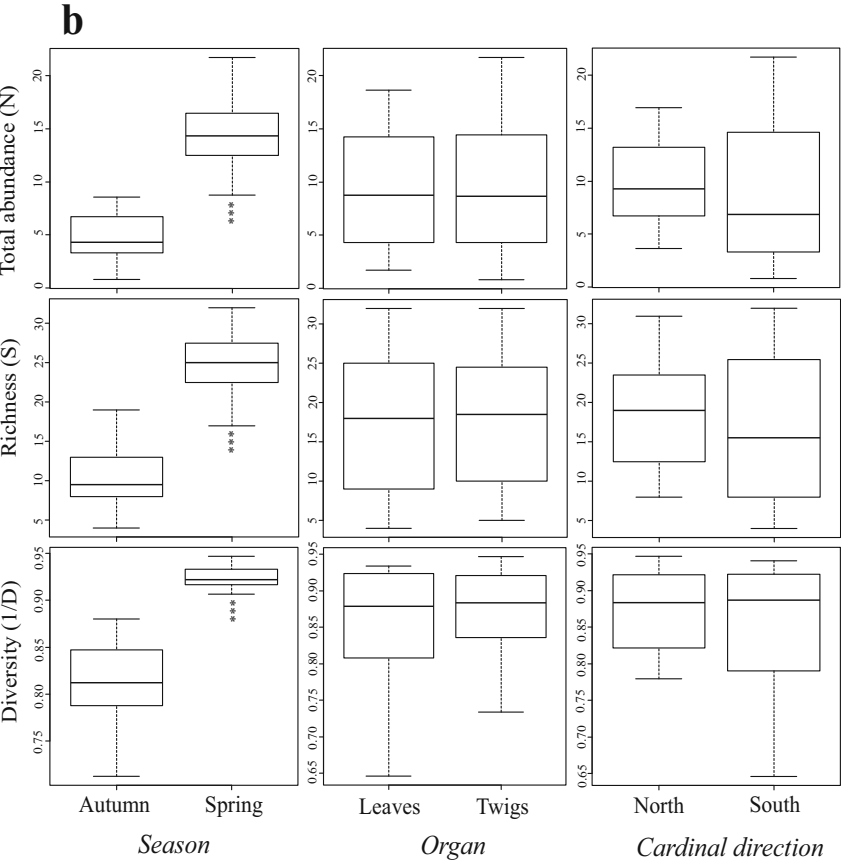

(autumn vs. spring), plant organs (leaves vs. twigs), and cardinal direction (north vs. south)

Almost $49 \%$ of the total fungal taxa found were shared between north and south (Fig. S1d). Therefore, taxonomic composition of fungal communities inhabiting plant tissues located in north and south directions of the olive trees is not shown.

\section{Season Is the Most Important Parameter for Shaping the Fungal Microbiota}

Co-inertia analysis was performed to determine global similarity of fungal community structures observed in each season, plant organ, and cardinal direction (Fig. 5). This analysis was also used to compute the contribution of each of these parameters to fungal structure and to identify fungal genera that contributed most to the total co-variance of samples. The structure of either endophytic $(F=8.47, P=0.001)$ or epiphytic $(F=51.15, P=0.001)$ communities was significantly affected by seasons (spring and autumn). In contrast, plant organ only had a significant effect on fungal endophytic composition ( $F=5.04, P=0.002)$, but not on epiphytic community $(F=1.74, P=0.122)$. Cardinal direction had no effect on both fungal community structures $(F=0.37$, $P=0.860$, for endophytes; $F=2.27 ; P=0.072$ for epiphytes). In fact, the majority of endophytic and epiphytic communities structure variations could be explained by season (12\% and $49 \%$, respectively), and in a lesser extent by plant organs (5\% and $0.7 \%$, respectively) and cardinal directions ( $0 \%$ and $1.2 \%$, respectively). By performing co-inertia analysis, seasons were confirmed to strongly affect the structure of both fungal communities (Fig. 5). Indeed, fungal community composition observed in spring was distinctly different from that observed in autumn, 

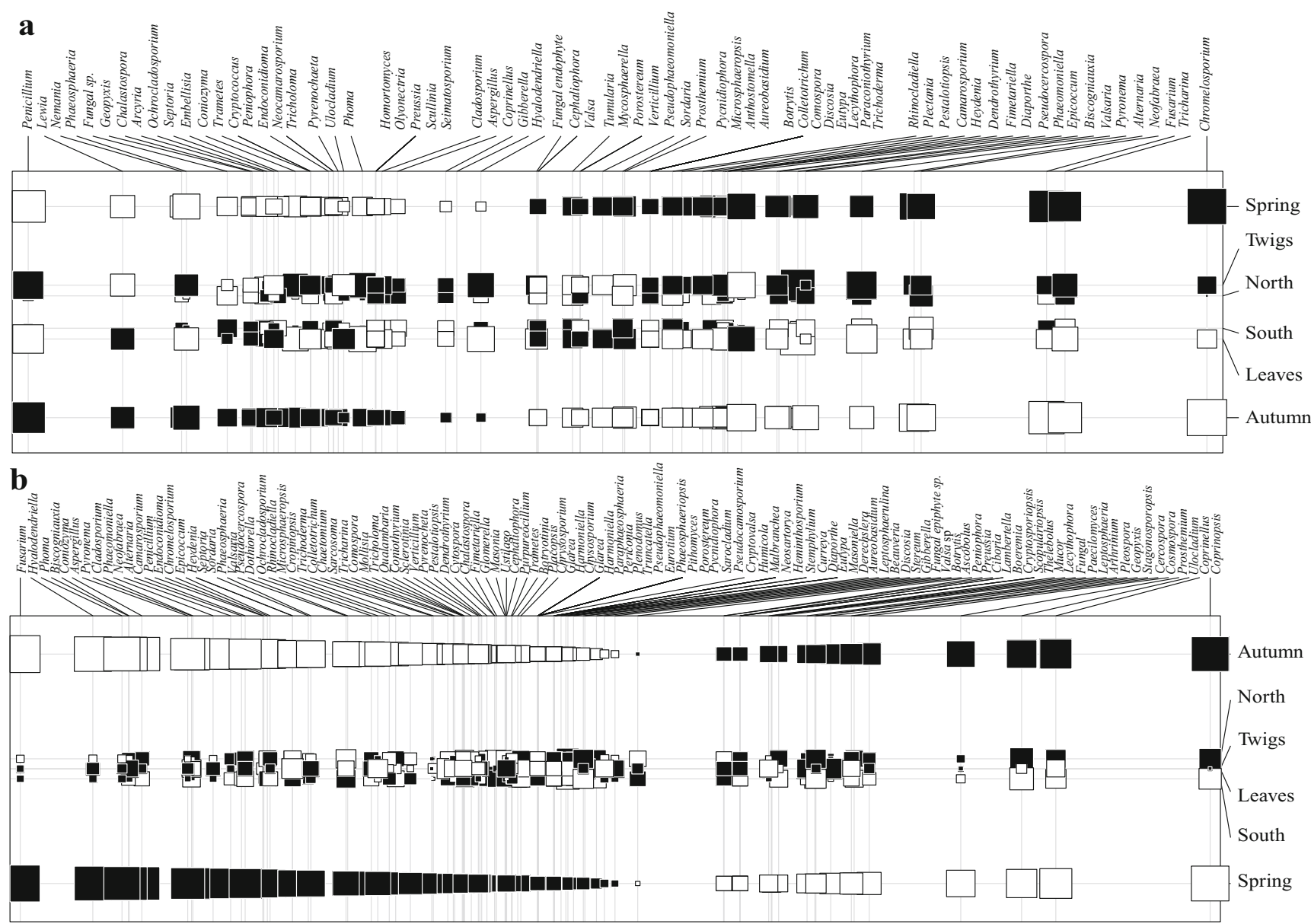

Fig. 5 Co-inertia factorial map showing positive (ם) and negative ( $\square$ ) relationships between endophytic (a) or epiphytic (b) fungal communities and seasons (spring vs. autumn), olive tree organs (leaves vs. twigs), and cardinal direction (north vs. south). Symbol sizes represent correlation strength. Distance between parameters represents their contribution towards discrimination of fungal community structures mainly due to the presence/abundance of certain genera. The endophytes Chromelosporium, Tricharina, Fusarium, Neofabraea, and Pyronema, and epiphytes Fusarium, Hyalodendriella, Phoma, Biscogniauxia, and Coniozyma were positively correlated with spring. The endophytes Penicillium, Lewia, Nemania, and Phaeosphaeria, and epiphytes Coprinopsis, Coprinellus, and Ulocladium were positively correlated with autumn. Although not so evident as for the season, both plant organs (twigs and leaves) also slightly influenced the fungal endophytic composition. Pyronema, Biscogniauxia, and Penicillium endophytic genera were positively correlated with twigs, while Pseudocercospora endophytic genus was positively correlated with leaves.

\section{Microclimate Conditions Affect Fungal Community Structures, Mainly the Epiphytic}

Our results indicate that the composition of phyllosphere fungi was greatly affected by seasons. This suggests that these microbial communities could be affected by climatic conditions. Using distance-based linear models (DistLM), the effect of several microclimate variables (mean temperature, maximum and minimum relative humidity, cumulative rainfall, and mean wind speed) on fungal assemblage structure was assess. To perform this analysis, weather data was collected 3-, 5-, 10- and 20-days before the sampling date. The results of marginal tests indicate that all climatic variables individually had a significant effect on endophytic and particularly on epiphytic community structures (Table 1). Sequential tests revealed that wind speed $(9.3 \%)$ caused the greatest amount of variation within epiphytic community, especially when occurring 5 to 20 days before sampling date (8.9\%, Tables 1 and S3). Variation was also caused by mean temperature $(4.8 \%)$, mainly when considering 20 days before sampling date $(0.6 \%)$, followed by cumulative rainfall $(4.2 \%)$, which occurred 3,10 , and 20 days before sampling date.

Variation of endophytic community structure was caused by rainfall $(6.3 \%)$, especially occurring from 5 to 20 days before sampling date $(6.1 \%$, Tables 1 and S3). Other factors that caused variation were mean temperature $(3.4 \%$, mainly when occurring 10 days before sampling date), wind speed (1.4\%, mainly when occurring 5 to 10 days before sampling date), and maximum relative humidity occurring 5 days before sampling $(0.9 \%)$. 
Table 1 Results of distance based linear model (DistLM) using fungal endophytic/epiphytic data and (A) each climatic variable (ignoring other variables) or (B) stepwise selection of variables, where the amount of variation explained by each variable added to the model is conditional on variables already considered

\begin{tabular}{llrll}
\hline A) Marginal tests & Climatic variable & \multicolumn{1}{l}{ F } & \multicolumn{2}{l}{ Prop. (\%) } \\
Epiphytic fungi & Mean temperature $\left({ }^{\circ} \mathrm{C}\right)$ & 9.92 & 0.001 & 7.37 \\
& Max. relative humidity $(\%)$ & 10.01 & 0.001 & 7.42 \\
& Min. relative humidity (\%) & 10.94 & 0.001 & 8.06 \\
& Cumulative rainfall $(\mathrm{mm})$ & 8.17 & 0.001 & 6.15 \\
& Mean wind speed $(\mathrm{m} / \mathrm{s})$ & 12.79 & 0.001 & 9.30 \\
Endophytic fungi & Mean temperature $\left({ }^{\circ} \mathrm{C}\right)$ & 7.69 & 0.001 & 5.81 \\
& Max. relative humidity $(\%)$ & 3.20 & 0.001 & 2.50 \\
& Min. relative humidity $(\%)$ & 6.54 & 0.001 & 4.98 \\
& Cumulative rainfall $(\mathrm{mm})$ & 8.41 & 0.001 & 6.31 \\
B) Sequential tests & Mean wind speed $(\mathrm{m} / \mathrm{s})$ & 3.51 & 0.001 & 2.74 \\
Epiphytic fungi & Climatic variable & $F$ & $P$ & Prop. $(\%)$ \\
Endophytic fungi & Mean temperature $\left({ }^{\circ} \mathrm{C}\right)$ & 6.94 & 0.001 & 4.82 \\
& Mean wind speed $(\mathrm{m} / \mathrm{s})$ & 12.80 & 0.001 & 9.30 \\
& Mean temperature $\left({ }^{\circ} \mathrm{C}\right)$ & 4.60 & 0.001 & 3.36 \\
\hline
\end{tabular}

$F$, statistic; $P$, probability; Prop., proportion of total variation explained

\section{Discussion}

This study provided significantly new insights of endophytic and epiphytic fungal communities associated with olive tree leaves and twigs, and the way they could be modulated by different biotic and abiotic factors. The fungal community of olive tree phyllosphere was found to be highly diverse (290 OTUs), supporting numerous genera (149) and families (68). Using a metabarcoding approach, Abdelfattah et al. [32], have previously revealed a rich fungal community (195 OTUs) associated with leaves, flowers and fruits of olive trees growing in a Mediterranean ecosystem (Italy). Using an approach similar to our study, 13 endophytic fungal taxa were found in the leaves and twigs of olive trees growing in another Mediterranean ecosystem (Portugal) [19]. However, epiphytic and endophytic fungal communities were not compared in these studies. Epiphytes inhabiting phyllosphere are under a set of selective pressures, which are distinct from those that endophytes are facing [12]. However, the implications of such differences have not yet been properly explored.

In our study, epiphytic community was found to be significantly richer and more abundant than endophytic community, as described for the phyllosphere of other woody plant systems $[14,15,33]$. On the surface of olive tree leaves and twigs, the composition of fungal communities was completely different from that in internal olive tissues. In previous studies, the same pattern was observed in leaves [13, 15] and stems [33] of other plant species. Epiphytic community was dominated by fungal species with melanized hyphae/spores belonging to the families Davidiellaceae (mostly Cladosporium spp.), Pleosporaceae (mostly Alternaria spp. and Ulocladium spp.), and Psathyrellaceae (mostly Coprinellus spp. syn. Coprinus spp.). In endophytic community, these species were found to be less abundant than non-pigmented fungal groups. Melanin is considered to confer tolerance to environmental stresses, such as UV radiation and desiccation [34]. Melanin accumulation seems to be advantageous in areas with high levels of radiation and extreme temperatures, such as Mediterranean regions [35]. Thus, the enrichment of melanized fungal groups on leaves and twigs surface of olive tree is likely driven by environmental climatic conditions.

The difference between surface and inner tissues is evident by the small fraction (32\%) of identified OTUs shared between both fungal communities. A similar conclusion was reached by Kharwar et al. [36] in Eucalyptus leaves, and by Kembel and Mueller [35] in greenbrier (Smilax rotundifolia) stems. As both endophytic and epiphytic communities had been simultaneously evaluated, our results suggest that endophytes outcompeted other fungi for colonization of leaves/ twigs or that host plant selects for fungal species or both. We hypothesize that phyllosphere fungal endophytes could have been epiphytes that penetrated into plant tissues $[11,36]$. This hypothesis is also reinforced by the low similarity found between endophytic and epiphytic communities composition on twigs when compared to leaves. Compared to twigs, leaves are more prone to be infected by epiphytes due to their extensive hairy surface, tenderness and presence of natural opening points (e.g., stomata). On olive tree phyllosphere, $38 \%$ of fungi were confined only to internal tissues of host plant. This suggests that they probably have originated from the soil and migrated through roots to aerial parts. Indeed, soil has been previously proposed as a potential endophytic inoculum for aboveground organs of grapevine [37].

Few studies have determined the influence of environmental and biological factors on the fungal composition of phyllosphere in Mediterranean crops, particularly when all 
these factors are simultaneously considered. In our study, season (spring vs. autumn) was the main factor shaping fungal community composition of olive tree phyllosphere. Likewise, seasonal variations were found to affect fungal endophytic communities in phyllosphere of other plant species growing in tropical (such as Tectona grandis; [38]), temperate (such as Pinus spp.; [39]), and in Mediterranean (such as Quercus ilex; $[4,40])$ areas. A similar effect was reported within epiphytic fungal community inhabiting the leaf surface of Camellia japonica [15] or Q. ilex [40], growing in temperate and Mediterranean areas, respectively. As expected, seasonality had greater influence on epiphytic than on endophytic fungal community structure. Furthermore, seasonal shifts were found to be partly related to climatic factors, as previously observed in fungal phyllosphere of $Q$. ilex in a mixed Mediterranean forest [40]. Unlike epiphytes, endophytes are confined within host plant tissues; therefore, they are likely to be less exposed to rapid changes in external environment [14]. In our study, wind speed (for epiphytic) and rainfall (for endophytic) occurring 5 to 20 days before sampling date were the climatic variables that better explain total variation of community structures. Thus, wind might be important for dispersal of epiphytic propagules [as reviewed by 41] and could be responsible for increasing diversity of epiphytic community. On the other hand, as rainfall mainly contributed for shaping endophytic community, rainfall might be important for fungal spore's dispersion and colonization of endophytes [as reviewed by 41]. In fact, rainfall has been previously identified as a key factor for the colonization pattern of endophytes [6]. Fungal community structure was also driven by mean temperatures at 10 (for endophytes) or 20 (for epiphytes) days before sampling date. At moderate temperatures (average temperature of $14.4^{\circ} \mathrm{C}$ in spring and $8.1{ }^{\circ} \mathrm{C}$ in autumn), fungal propagules would have higher viability, thus increasing successful colonization of plant tissues [4]. Therefore, the highest abundance and richness of fungi observed during spring could be due to the warmer temperatures and higher wind speed (for epiphytes) or rainfall (for endophytes) observed during this season in comparison with autumn. Similarly, other studies have reported that richness and abundance of fungal endophytes [4] and epiphytes [15] was higher in spring than in autumn. All these findings suggest that seasonal shifts among phyllospheric fungi may be due to climatic conditions that favor propagule production or dispersal and/or to different abilities exhibited among fungal species. For example, spring preference of sporulation and differential capacity of fungi to adapt to climate have been described by Vacher et al. [42] or Martinez-Alvarez et al. [6]. Indeed, a great number of endophytic and epiphytic fungal genera were found to be positively associated with either spring or autumn.

Olive plant organ was found to influence the endophytic fungal composition, but not the epiphytic. To the best of our knowledge, no previous studies have investigated the specificity of epiphytic fungi towards different host organs. In this study, colonization and composition of fungal community on the surface of twigs and leaves was not greatly different, suggesting no organ-specificity among epiphytes. This is not surprising because the canopy of woody evergreen plants, such as olive trees, is exposed to the same aerial inoculum. Both plant organs are reasonably close to each other, so they may even be in direct contact with each other, leading to a high number of shared OTUs. In what concerns endophytes, our study demonstrates that twigs harbor more species and strains than leaves, as described for other plant systems growing in Mediterranean climate, such as Quercus spp. and Pinus spp. $[4,6,7]$. On the other hand, in tropical trees the endophytic fungal richness in leaves was reported to be higher [38] or lower [43] when compared to stems. Although endophytic composition was similar in leaves and twigs, several endophytic fungal genera were found to be more correlated either with leaves or twigs, suggesting signs of organ specificity by certain genera. This hypothesis is also supported by the fact that a higher number of endophytes are observed in twigs than in leaves. As leaves are soft tissues, they are more exposed to external environment than woody twigs. Therefore, climatic factors influence more the endophytic community on leaves than twigs, resulting in the lower endophyte richness found in leaves. This result is in agreement with previous studies indicating the possible organ-specificity of fungal endophytes (e.g., $[4,7,44])$.

Exposure to ultraviolet radiation depends on organ orientation (north or south) within canopies and is expected to influence the colonization of epiphytic and endophytic phyllosphere fungi [45]. However, our results revealed no significant differences in the diversity and composition of fungal communities inhabiting leaves or twigs at different canopy locations (north and south). Few studies have compared phyllospheric fungi inhabiting plant organs oriented in north and south directions. In the phyllosphere microbiota of beech (Fagus crenata), Osono and Mori [46] reported that the composition and richness of species inhabiting shade leaves differed from that of sun leaves. They detected that endophytes and epiphytes showed higher richness in sun and shade leaves, respectively. In contrast, Laforest-Lapointe et al. [45] found that canopy location did not have a significant influence on the epiphytic bacterial community structure in leaves of several temperate forest tree species, including angiosperms and gymnosperms. Although phyllosphere fungi did not differ significantly with respect to the location of plant organs that they inhabit, we found that both canopy location and season jointly affected the diversity and abundance of fungal phyllosphere community. In spring, fungal endophytes were more affected by canopy location than epiphytes. An opposite pattern was observed in autumn, being epiphytes more susceptible to the effect of canopy location. Therefore, although canopy orientation does not have a great influence on fungal 
assemblage, it does have a more pronounced effect on fungal epiphytic and endophytic communities during autumn and spring, respectively.

In conclusion, endophytic and epiphytic fungal communities of olive tree phyllosphere are distinct and are not driven by the same factors. While epiphytic fungal assemblage appeared to be largely influenced by environmental factors (mostly season, but also wind speed and mean temperature), endophytic fungal assemblage is driven by both environmental factors (mostly season and slightly by rainfall and mean temperature) and plant organs. The potential or ecological significance of both phyllosphere fungal communities is unknown, and further investigations are still required to identify the functional role of these microorganisms.

Acknowledgements This work is funded by FEDER funds through COMPETE (Programa Operacional Factores de Competitividade) and by national funds by FCT (Fundação para a Ciência e a Tecnologia) within the framework of the project EXCL/AGR-PRO/0591/2012. T. Gomes thanks FCT, POPH-QREN, and FSE for PhD SFRH/BD/98127/ 2013 grant.

\section{Compliance with Ethical Standards}

Conflict of Interest The authors declare that they have no conflict of interest.

Ethical Approval This article does not contain any studies with human participants or animals performed by any of the authors.

\section{References}

1. Lindow SE, Brandl MT (2003) Microbiology of the phyllosphere. Appl Environ Microbiol 69:1875-1883

2. Berg G, Grube M, Schloter M, Smalla K (2014) Unraveling the plant microbiome: looking back and future perspectives. Front Microbiol 5:148

3. Moricca S, Ragazzi A (2008) Fungal endophytes in Mediterranean oak forests: a lesson from Discula quercina. Phytopathology 98 : 380-386

4. Collado J, Platas G, González I, Peláez F (1999) Geographical and seasonal influences on the distribution of fungal endophytes in Quercus ilex. New Phytol 144:525-532

5. Linaldeddu BT, Sirca C, Spano D, Franceschini A (2011) Variation of endophytic cork oak-associated fungal communities in relation to plant health and water stress. For Pathol 41:193-201

6. Martinez-Alvarez P, Martin-Garcia J, Rodriguez-Ceinos S, Diez JJ (2012) Monitoring endophyte populations in pine plantations and native oak forests in Northern Spain. For Syst 21:373-382

7. Moricca S, Ginetti B, Ragazzi A (2012) Species- and organspecificity in endophytes colonizing healthy and declining Mediterranean oaks. Phytopathol Mediterr 51:587-598

8. Botella L, Santamaría O, Díez JJ (2010) Fungi associated with the decline of Pinus halepensis in Spain. Fungal Divers 40:1-11

9. Martín-García J, Espiga E, Pando V, Diez JJ (2011) Factors influencing endophytic communities in poplar plantations. Silva Fenn 45:169-180

10. Rodriguez RJ, White JF, Arnold AE, Redman RS (2009) Fungal endophytes: diversity and functional roles. New Phytol 182:314-330
11. Porras-Alfaro A, Bayman P (2011) Hidden fungi, emergent properties: endophytes and microbiomes. Annu Rev Phytopathol 49:291-315

12. Rastogi G, Coaker GL, Leveau JH (2013) New insights into the structure and function of phyllosphere microbiota through highthroughput molecular approaches. FEMS Microbiol Lett 348:1-10

13. Osono T (2007) Endophytic and epiphytic phyllosphere fungi of red-osier dogwood (Cornus stolonifera) in British Columbia. Mycoscience 48:47-52

14. Santamaria J, Bayman P (2005) Fungal epiphytes and endophytes of coffee leaves (Coffea arabica). Microb Ecol 50:1-8

15. Osono T (2008) Endophytic and epiphytic phyllosphere fungi of Camellia japonica: seasonal and leaf age-dependent variations. Mycologia 100:387-391

16. Loumou A, Giourga C (2003) Olive groves: "the life and identity of the Mediterranean". Agric Hum Values 20:87-95

17. SNIRH (2016) http://snirh.apambiente.pt. Accessed 22 Aug 2016

18. Malavolta C, Perdikis D (2012) Guidelines for integrated production of olives. IOBC technical guideline III, vol 77. 2nd edn, pp 1-19

19. Martins F, Pereira JA, Bota P, Bento A, Baptista P (2016) Fungal endophyte communities in above- and belowground olive tree organs and the effect of season and geographic location on their structures. Fungal Ecol 20:193-201

20. White TJ, Bruns T, Lee S, Taylor J (1990) Amplification and direct sequencing of fungal ribosomal RNA genes for phylogenetics. In: Innis MA, Gelfand DH, Sninsky JJ, White TJ (eds) PCR protocols: a guide to methods and applications. Academic Press, New York, pp 315-322

21. Oliveira I, Pereira JA, Lino-Neto T, Bento A, Baptista P (2012) Fungal diversity associated to the olive moth, Prays oleae Bernard: a survey for potential entomopathogenic fungi. Microb Ecol 63:964-974

22. Seaby RM, Henderson PA (2006) Species diversity and richness version 4. Pisces Conservation, Lymington

23. Colwell RK (2013) EstimateS: statistical estimation of species richness and shared species from samples, Version 9. http://purl.oclc. org/estimates. Accessed 7 Jan 2016

24. R Core Team (2014) R: a language and environment for statistical computing. http://www.R-project.org/. Accessed 22 Mar 2016

25. Zuur AF, Ieno EN, Walker NJ, Saveliev AA, Smith GM (2009) Mixed effect models and extensions in ecology with R. Springer, Berlin

26. Magurran AE (2004) Measuring biological diversity2nd edn. Blackwell Science Ltd, Oxford

27. Bray JR, Curtis JT (1957) An ordination of upland forest communities of southern Wisconsin. Ecol Monogr 27:325-349

28. Clarke KR, Gorley RN (2015) PRIMER v7: user manual/tutorial. PRIMER-E, Plymouth

29. Henderson PA, Seaby RMH (2007) Community analysis package 4.0. Pisces Conservation Ltd, Lymington

30. Dolédec S, Chessel D (1994) Co-inertia analysis: an alternative method for studying species environment relationships. Freshw Biol 31:277-294

31. Mcardle BH, Anderson MJ (2001) Fitting multivariate models to community data: a comment on distance-based redundancy analysis. Ecology 82:290-297

32. Abdelfattah A, Nicosia MGLD, Cacciola SO, Droby S, Schena L (2015) Metabarcoding analysis of fungal diversity in the phyllosphere and carposphere of olive (Olea europaea). PLoS One 10:e0131069

33. Zambell CB, White JF (2014) In the forest vine Smilax rotundifolia, fungal epiphytes show site-wide spatial correlation, while endophytes show evidence of niche partitioning. Fungal Divers 75: 279-297

34. Gessler NN, Egorova AS, Belozerskaya TA (2014) Melanin pigments of fungi under extreme environmental conditions (review). Appl Biochem Microbiol 50:105-113 
35. Kembel SW, Mueller RC (2014) Plant traits and taxonomy drive host associations in tropical phyllosphere fungal communities. Botany 92:303-311

36. Kharwar RN, Gond SK, Kumar A, Mishra AA (2010) A comparative study of endophytic and epiphytic fungal association with leaf of Eucalyptus citriodora Hook., and their antimicrobial activity. World J Microbiol Biotechnol 26:1941-1948

37. Zarraonaindia I, Owens SM, Weisenhorn P, West K, HamptonMarcell J, Lax S, Bokulich NA, Mills DA, Martin G, Taghavi S et al (2015) The soil microbiome influences grapevine-associated microbiota. MBio 6:e02527-e02514

38. Singh DK, Sharma VK, Kumar J, Mishra A, Verma SK, Sieber TN, Kharwar RN (2017) Diversity of endophytic mycobiota of tropical tree Tectona grandis Linn. f.: spatiotemporal and tissue type effects. Sci Rep 7:3745

39. Kim C, Eo J, Eom A (2013) Diversity and seasonal variation of endophytic fungi isolated from three conifers in Mt. Taehwa, Korea. Mycobiology 41:82-85

40. Peñuelas J, Rico L, Ogaya R, Jump AS, Terradas J (2012) Summer season and long-term drought increase the richness of bacteria and fungi in the foliar phyllosphere of Quercus ilex in a mixed Mediterranean forest. Plant Biol 14:565-575
41. Vacher C, Hampe A, Porte AJ, Sauer U, Compant S, Morris CE (2016) The phyllosphere: microbial jungle at the plant-climate interface. Annu Rev Ecol Evol Syst 47:1-24

42. Jumpponen A, Jones KL (2010) Seasonally dynamic fungal communities in the Quercus macrocarpa phyllosphere differ between urban and nonurban environments. New Phytol 186: 496-513

43. Verma SK, Gond SK, Mishra A, Sharma VK, Kumar J, Singh DK, Kumar A, Goutam J, Kharwar RN (2014) Impact of environmental variables on the isolation, diversity and antibacterial activity of endophytic fungal communities from Madhuca indica Gmel. at different locations in India. Ann Microbiol 64:721-734

44. Sun X, Ding Q, Hyde KD, Guo LD (2012) Community structure and preference of endophytic fungi of three woody plants in a mixed forest. Fungal Ecol 5:624-632

45. Laforest-Lapointe I, Messier C, Kembel SW (2016) Tree phyllosphere bacterial communities: exploring the magnitude of intra- and inter-individual variation among host species. Peer $\mathrm{J} 4$ : e2367

46. Osono T, Mori A (2003) Colonization of Japanese beech leaves by phyllosphere fungi. Mycoscience 44:437-441 